\title{
EEICCP_Energy Efficient Protocol for Wireless Sensor Networks
}

\author{
Shalli Rani ${ }^{{ }^{*}}$, Jyoteesh Malhotra ${ }^{2}$, Rajneesh Talwar ${ }^{3}$ \\ ${ }^{1}$ SSD Women's Institute of Technology, Bathinda, India \\ ${ }^{2}$ ECE, GNDU Regional Centre, Jalandhar, India \\ ${ }^{3}$ ECE, SWIFT Technical Campus, Patiala, India \\ Email: *Shavi_jindal@yahoo.com, jyoteesh@gmail.com, rtphdguidance@gmail.com
}

Received May 22, 2013; revised June 4, 2013; accepted June 12, 2013

Copyright (C) 2013 Shalli Rani et al. This is an open access article distributed under the Creative Commons Attribution License, which permits unrestricted use, distribution, and reproduction in any medium, provided the original work is properly cited.

\begin{abstract}
In this paper, an energy efficient inter cluster coordination protocol developed for the wireless sensor networks has been proposed. By controlling the topology, longevity and the scalability of the network can be increased. Clustering sensor node is an effective topology for the energy constrained networks. So cluster based algorithm has been developed in which different levels of clusters are considered on the basis of received signal strength to recognize the distance of the clusters from the BS (base station) and to determine the number of cluster coordinators to make routes for the CHs to transmit the data. Based on the investigation of existing protocols in which cluster heads send data directly to the base station, it is found that direct transmission by the CHs is not an optimal solution and dissipates a lot of energy, so in this paper a novel EEICCP (Energy efficient inter cluster coordination) protocol has been proposed which evenly distributes the energy load among the sensor nodes and use the multi hop approach for the CHs. Analytical model of new protocol is projected and the algorithm is implemented in MATLAB. Moreover, EEICCP has shown remarkable improvement over already existing LEACH and HCR protocols in terms of reliability and stability. Our work has also been validated through the simulation results.
\end{abstract}

Keywords: HCR; LEACH; EEICCP; Transfer and Election Phase

\section{Introduction}

The need of the wireless sensor network (WSN) came into existence with the military applications which is now used in environmental monitoring, health care applications, industry process control, security applications etc. Sensors can collect the data about the temperature, humidity and moving objects, which are battery operated and can't be recharged after deployment. This limitation of sensors leads to the failure of the network when their energy is depleted, affecting the reliability of the network. Major concern of WSN is how to collect data from the densely deployed nodes, in the heterogeneous or the homogenous way. In the heterogeneous network, some nodes are having more energy than the other nodes [1] because they act as the relay nodes, but in the homogenous networks all nodes have same energy level, proposed protocol EEICCP (Energy Efficient Inter Cluster Coordination Protocol) uses homogenous type of nodes. In the homogeneous cluster based network the cluster

"Corresponding author. heads as in HCR, LEACH [2,3] are randomly chosen to perform the duty of the relay nodes to transfer, data and after sometime cluster heads exchange their roles with normal nodes for the consumption of uniform energy of all the nodes. Same technique is adopted in the EEICCP but with variations in the number of iterations that are less in proposed protocol and which reduce the complexity as well. Network can become obsolete if nodes lose their battery power up to the extent when it is less than the threshold level of the energy, it ensues because sensors are battery operated and they have limited battery power.

To deal with the problems of energy consumption in WSN, the researchers have used many techniques in which they have suggested that cluster heads $(\mathrm{CH})$ should be chosen $[2,3]$ to forward the data of the nodes. Role of the $\mathrm{CH}$ is rotated to deplete energy with the same extent. But in these techniques, all the cluster heads send their data directly to the base station which increases energy consumption. In HCR [3] the associative cluster heads $(\mathrm{ACH})$ are elected to exchange the role of the cluster heads but even in that case $\mathrm{CH}$ sends data directly 
to the base station which consumes a lot of energy due to the multipath channel fading [4] and increases the path loss exponent. To deal with the problem of path loss exponent, EEICCP uses line of sight propagation which reduces the value of the exponent and hence energy consumption. A distributed beamforming is used in this protocol to force the transfer of data in the desired direction as explained in [5]. The protocol discussed in this paper uses homogenous dense network and multi-hop routing where the cluster heads and the cluster coordinators (CCO) are elected in the same way as $\mathrm{CH}$ in the $\mathrm{LEACH}$ [2] and ACH in the HCR [3], but the cluster coordinators in each cluster will vary according to the depth (means according to the distance from the base station). According to the levels of the clusters, the parameter of depth decides the number of CCO's in EEICCP which is considered in the same way as it is defined in tree based data structure algorithms where the leaf nodes (nodes which have no child further) are at the maximum depth. The cluster c1 (Figure 1) is well thought-out to be as the leaf cluster which has no CCO. This strategy has shown improvement over the LEACH and HCR [2,3] in terms of complexity, energy consumption and reliability (Quality of service (QoS) metrics) in election phase, transfer phase etc. Sensor nodes have a lot of data to be processed by the human operator or automatically. Data can be aggregated by data fusion which can combine many data measurements for the accurate signal by reducing the SNR. Task of aggregation and data fusion is application specific as acoustic signals are combined through the beam forming approach. A lot of energy can be conserved by locally executing the algorithm of data fusion and data aggregation. Information which is collected on the sink can be of different types like normal data, real time data etc. So, a design is needed for the information aware routing with efficient transmission. This strategy will be considered in our next research work.

\section{Paper Organization}

The rest of this paper is organized as follows. Section 2 provides the brief introduction of the related work. Mathematical model used by EEICCP protocol has been elaborated in Section 3. Overview of the new algorithm and the EEICCP protocol has been outlined in the Section 4. Energy consumption in all the phases of the proposed strategy and the derived equations used in the various simulations are discussed in the Section 5 . Section 6 explains the results and validation of the protocol based on the QoS metrics before the conclusion in the Section 7.

\section{Related Work}

There are many emerging applications out of which en- vironment monitoring application is very important and very popular. It needs densely deployed network which has lead to the development of energy efficient and QoS based protocols. Still there is a lot to be done to entirely exploit the benefits of wireless sensor networks. SEP (Stable Election Protocol) works for the election of cluster heads in a distributed fashion in two-level hierarchical wireless sensor networks. Election of nodes as cluster heads depends upon the starting energy of the nodes because some nodes are more powerful than the others in contrast to newly protocol (EEICCP) as energy is same for all the nodes so any node can be the cluster head in starting and it is elected randomly like LEACH [2] and the HCR [3] Longevity of the network is increased by using EEICCP because energy consumption is very less in this protocol. To solve the hot spot problem an Unequal Cluster-based Routing (UCR) protocol [6] is devised. In technical terms the hotspot problem can be described as the isolation of the sink node from the rest of the network as a result of the power exhaustion of nodes in the hotspot area and the area in the interior of the maximum transmission of the sink node is the hotspot area. Hot spot problem arises when approximately all the data is transferred from the nodes which are nearest to the sink. Those nodes bear the load of all other nodes and hence deplete their energy earlier than others which results in the death of nodes and make it difficult for the other nodes to transfer the data as with the death of nodes near to the sink will end the routing path to the sink hence network will become archaic. This problem does not arise in EEICCP because the role of $\mathrm{CH}$ and CCO is rotated after one round of transferring data. One round gets accomplished when the data of all the nodes of all the clusters is sent. UCR groups the nodes into clusters of unequal sizes as contrast to EEICCP in which all clusters have uniform size assumed of 100 nodes. In UCR Cluster heads closer to the base station have smaller cluster sizes than those farther from the base station, to save some energy for the inter-cluster communication to forward the data. This is not needed in EEICCP because there is always one CCO in each cluster (low distance from BS) above the lower cluster (higher distance from BS) which makes it less complex than the UCR. Clustering can save lot of energy comparative to non cluster organization which is the flat architecture [7]. Many energy efficient algorithms have been developed and they have proved that clustering has proved best in saving energy so cluster organization is adopted in EEICCP. Some protocols have to be determined how to select the cluster head in optimal way and what should be the cluster size [8]. But cluster size is assumed to be static in EEICCP protocol to achieve long life for the WSN. Different techniques for the election of cluster head came into existence like coverage metric of node [9], making the protocol more com- 
plex and needs more processing making it less suitable for the accurate results and making it less reliable comparative to the EEICCP which is simple in election process of CH like LEACH and HCR [2,3]. Cluster heads are constant in EEICCP but they will exchange their role with normal nodes after every round i.e. they will be elected after every round, a different approach from EECS [10] where election of cluster heads is constant and in one round only .It makes the uniform distribution and consumption of energy in EEICCP serving in the longevity of the WSN. In EECS [10] the communication between base station and the cluster head is direct like other algorithms as LEACH, HCR [2,3] by which more energy is consumed. New protocol has given the solution of this problem described in Section 6. In EECHA [11] the back up of the first cluster head has been maintained by making two types of cluster heads primary and secondary for the longevity of network but this is done through CCOs' in EEICCP which do not wait for the diminishing of the $\mathrm{CH}$ so processing is reduced in EEICCP.

Transmission of data to the base station is possible in two ways, hierarchical in which nodes are categorized into normal nodes and CHs' and in non-hierarchical way i.e. flat architecture [7] where all nodes play same role and act as peers. Hierarchical way is important for scalability feature including the communication among the nodes and the cluster heads, and also between cluster heads i.e. inter cluster co-ordination as adopted by newly devloped protocol. Deployment of nodes can be uniform like LEACH, HCR [2,3] and non-uniform distribution as in UCR [6]. Different design aspects like heterogeneity where some nodes $(\mathrm{CH})$ are made more powerful by providing more energy to act as relays [1] and homogeneity where all nodes have same level of energy [2,3] to achieve same level of energy consumption, have been implemented for various applications. The hierarchical homogenous design has been used for new proposed protocol.

\section{Our Contribution and Problem Definition}

Wireless sensor network is energy constrained because of the limited battery power of the sensors. In cluster based single hop conventional protocols when data is sent directly to base station, value of path loss exponent is increased due to multipath fading channel [4,12] which affects the rate of energy consumption, directly proportional to it, same happens in LEACH [2] and HCR [3]. So a new protocol EEICCP (energy efficient inter cluster coordination protocol) has been developed where deployment of nodes is homogenous, transmission of data is multi hop and it uses the line of sight propagation to reduce the path loss exponent to reduce the energy consumption. Variation of CCOs' in each cluster according to the distance of the cluster from the base station has not been considered till now in any research work which is taken in EEICCP protocol and various new equations have been derived from the mathematical model explained in Section 3 and new algorithm explained in Section 4 has been developed for inter cluster communication between the $\mathrm{CH}$ and $\mathrm{CCO}$ and the results of the simulations based on new equations have been discussed in Section 6 which has proved that by this protocol energy reduction up to large extent has been gained which will be useful in the long network life time.

\section{Mathematical Model}

Many of the research protocols have used the first order radio model as described in [2]. Energy is dissipated while transmitting and receiving the data and energy consumption for the short distance is $d^{2}$ when propagation is in line of sight and $d^{4}$ for the long distance due to multipath fading propagation $[4,12]$. It works on the routine measurements and sensing takes place constantly resulting in steady volume of data being transmitted to the sink. In future implementation of the query driven and the event driven simulation will be considered. The following assumptions have been made in EEICCP protocol:

1) Base station is fixed; sensors are densely deployed and are static. Number of clusters is predetermined for the WSN. They will pass the data on the predefined path in which clusters, the CHs and the CCOs are numbered according to their distance (depth) based on received signal strength (RSS) and the data passes according to the newly developed algorithm (Section 4, Figure 1).

2) Some sensors are far away from the base station (BS) that is why the cluster head will consume the $d^{4}$ loss of energy for transmitting 1 bit data in direct transmission. So data is passed through multiple hops and reach the BS by cluster very near to the base station which will have CCOs for all the CHs below it. Propagation from these CCOs' will be in line of sight and hence path loss will be $d^{2}$. So experiments accomplished by both the channel models, multipath fading ( $d^{4}$ path loss) and free space $\left(d^{2}\right.$ path loss), depending upon distance between transmitter and receiver [13]. This loss can be inverted by aptly setting the power amplifier if the distance is less than verge then free space otherwise multipath model is used.

3) Links are symmetric i.e. same level of power is required for the communication between any two nodes. Algorithm (Section 4, 4.2) developed to transfer the data to the BS is non adaptive i.e. it does not consider any changes in the topologies and the load.

4) Nodes transmit data to the $\mathrm{CH}$ by TDMA schedule.

Geocost protocols, widely used in Ad-hoc and the Wireless sensors networks [4] mostly suffer from the issues of energy efficiency so they have to make the deployment of the sensors in that way that energy con- 


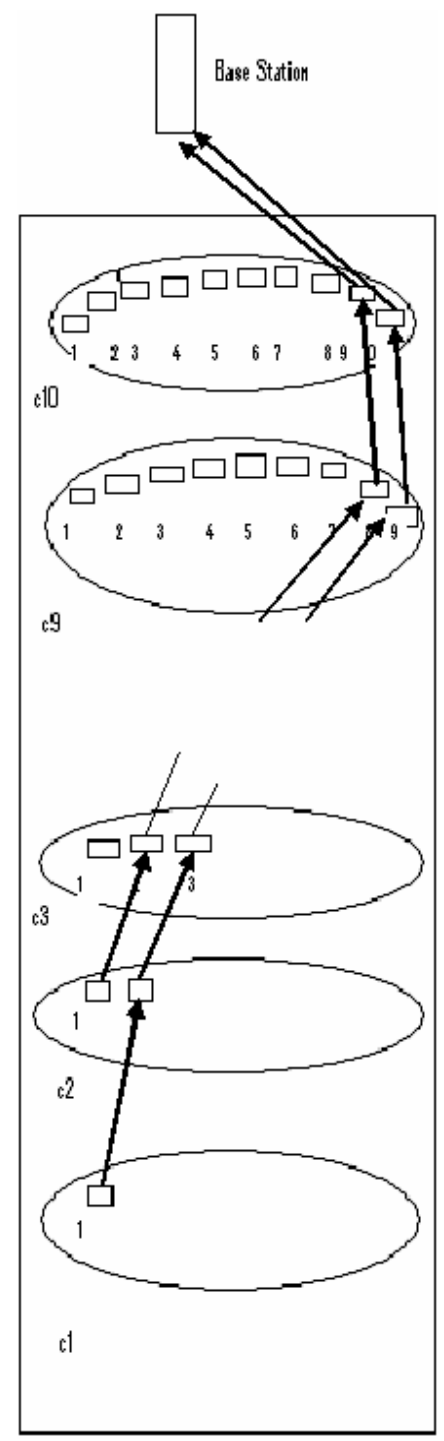

Figure 1. Transfer of data with the help of coordinators (Number $\mathbf{1}$ in every cluster is taken for $\mathbf{C H}$, and all others $(2,3 \cdots 10)$ act as CCOs).

sumption is minimum, in the same way the sensors are organized in this way that one cluster will always be very close to the base station. Energy is function of the inter nodes communication that is taken $d^{\mathrm{n}}$. A two ray model [14] describes the relation of the distance with received power which is inversely proportional to the distance $d$. So protocol EEICCP considers that if the distance is short then the path loss exponent is taken $d^{2}$ and if the distance is long then the path loss exponent will be $d^{4}$.

The energy consumed in transmission includes the data size and the distance, the energy consumption in receiving mode includes the data volume only [12]. The energy loss equations used in this protocol are:

$$
\begin{aligned}
& E_{T}=l \times E_{e}+l \times e l \times d^{4} \\
& E_{T}=l \times E_{e}+l \times e s \times d^{2}
\end{aligned}
$$

$$
E_{R}=l \times E_{e}+l \times E_{b f}
$$

$E_{T}$ is the energy used in transferring and $E_{R}$ is the energy consumed in receiving the data. Equation (1) is used when nodes have to send the data at the long distance and Equation (2) is used for the data transmission at short distance. $E_{b f}$ is the beam forming approach while recaption of data which reduces the energy consumption. The different values used for complete process are given in Table 1.

\section{EEICCP Protocol}

The protocol depends upon the fact that some cluster head sends data directly to the base station (Single hop) and the some send by multi hop transmission, but energy consumption in conventional protocols increases due to multi path fading channel which affects the network life time. An attempt has been made to reduce this power loss in to free space model that is $d^{2}$. The arrangements of the nodes has been done in this way that one cluster always, is very close to the base station i.e. in line of sight propagation and that cluster will have head nodes sufficient for all the below clusters which will forward the data of all those clusters. Layers of clusters have been formed so there is always one cluster coordinator for every lowest cluster. The division of clusters is done from top to bottom. EEICCP protocol works by starting the election phase in which the cluster heads are elected according to the distance based on RSS. Number of clusters is fixed so as the cluster heads and the cluster coordinators. After election of cluster coordinators by the CHs, a cluster id is assigned to each cluster head and the cluster coordinator. This id is transmitted by each cluster to their nodes by the advertisement message. And cluster co-coordinators also pass their own ids. After that the transmission phase begins in which data is transferred to the cluster head and that data is passed to the base station with the help of CCOs. In first round the data is collected by the $\mathrm{CH}$ of that cluster which has data to send, then in the other iterations the data is passed to the base station with the help of cluster co-coordinators. The path is set for the data transmission with the help of the cluster coordinators ids.

Table 1. Values used for the energy consumption in analytical model.

\begin{tabular}{ccc}
\hline Description & Symbol & Value \\
\hline $\begin{array}{c}\text { Energy consumed by the amplifier to } \\
\text { transmit at a short distance }\end{array}$ & Es & $10 \mathrm{pJ} / \mathrm{bit} / \mathrm{m}^{2}$ \\
$\begin{array}{c}\text { Energy consumed by the amplifier to } \\
\text { transmit at a long distance }\end{array}$ & El & $\begin{array}{c}0.0013 \\
\mathrm{pJ} / \mathrm{bit} / \mathrm{m}^{4} \\
\text { Energy consumed in the electronics circuit } \\
\text { to transmit or receive the signal }\end{array}$ \\
$\begin{array}{c}\text { Energy consumed for beam forming } \\
\text { E }\end{array}$ & $E_{e f}$ & $50 \mathrm{~nJ} / \mathrm{bit}$ \\
\end{tabular}




\subsection{Election Phase}

In the election phase the cluster heads and the cluster cocoordinators are elected. The energy of all the nodes is same. Heads are elected by the nodes and the co-coordinators are elected by the cluster head. The cluster head sends an advertisement message to the nodes and the nodes send acknowledge message to the cluster head. CH's in EEICCP act as local in charge of centers to coordinate the data transmissions in their clusters. Setting up of a TDMA schedule is accomplished by $\mathrm{CH}$ and which transmits this schedule to the nodes in the cluster. This ensures collision free transmission among data messages and also allow non-cluster heads to turn off the radio components of each node at all times except during their transmit time, and hence improve the energy reduction energy consumption by the nodes. After the knowledge of TDMA schedule by all nodes in the cluster, the initial phase is complete.

Algorithm of EEICCP protocol works in this way, that after the data transmission by all the nodes of all the clusters, one round gets complete and election phase restarts. The cluster heads are elected on the basis of the number of the clusters. As 1055 nodes are taken in simulation of newly protocol, dividing the 1000 nodes into ten clusters of 100 nodes each. As there are layers of clusters one above the other so the formula of sum of first $n$ natural numbers $(n(n+1) / 2)$ is used to calculate the $\mathrm{CHs}$ needed for all the 10 clusters $(n)$ and they are in increasing sequence starting from the depth first which has only one $\mathrm{CH}$ (with highest distance from the BS) and they are in the order of $(1,2, \cdots 10$, Figure 1) as movement is accomplished from depth to the BS, so cluster nearest to the BS has $10 \mathrm{CHs}$. In each cluster out of all the CHs in that cluster one is the $\mathrm{CH}$ for the nodes of that cluster and other are acting as the CCOs for the clusters below them.

\subsection{Data Transfer Phase}

Transmission begins from the depth first $\mathrm{CH}$ which goes to the CCO of the next higher level cluster and so on as shown in Figure 1 which is finally sent to the BS by the CCO of the last cluster nearest to the BS. In this way the distance travelled is decreased and the propagation of data is in the line of sight leading to the reduction in the path loss exponent.

At first time the data of cluster with the highest depth (farther away cluster from BS) is sent to the base station based on the predetermined path, then to the HD (Highest depth)-1 CCO and so on as shown in Figure 1 which describes the structural form of the inter cluster communication. In this diagram it can be seen that the c1 will send data to the cluster coordinator 2 of the cluster c2. That is forwarded to the cluster coordinator 3 of cluster c3. And in this way lastly it reaches to the $10^{\text {th }} \mathrm{CCO}$ of c10 cluster which will forward it to the base station. c10 cluster is very close to the base station so instead of taking the distance of multipath fading it uses the free space model. Energy in large extent has been reduced by using this approach discussed in Section 6. The Algorithm 1 shows the process of data transmission and its flow chart is given in Figure 2.

\section{Energy Consumption}

Energy is consumed in all the phases of protocol, in the starting for the election of the cluster heads. After election phase, data transmission phase starts in which firstly all the sensor nodes in their clusters send their data to their respective cluster heads so energy is consumed in sending the and receiving the data. Data travels with the help of cluster coordinators which again needs the power to send and receive the data until it reaches the BS. By doing simulation of this process the results show that by increasing the cluster heads, the energy consumed is increased than when the cluster heads are less which is very contrast to the HCR and LEACH [2,3]. In HCR [3] it is shown that energy consumed is less when there are more cluster heads, same is explained in LEACH [2] but proposed protocol shows different approach than both of these protocols. The energy consumed is equated through the following evaluated expressions:

1) Election phase: In election phase the cluster heads send advertisement message to the sensor nodes and the nodes reply with acknowledgment. The energy consumed by the cluster head in election phase evaluated through the Equations (2) and (3) by which Equation (4) is formulated:

$$
\text { Ech_elec }=l\left(E e+E s \cdot d^{2}\right)+n 1 \cdot l\left(E e+E_{b f}\right)
$$

The first part of Equation (4) shows the energy consumed in sending the message to the sensor nodes and the second part shows the energy consumed in receiving

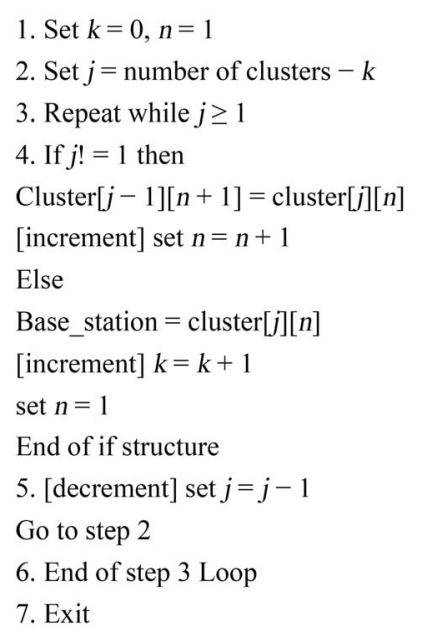

Algorithm 1. For data transmission. 


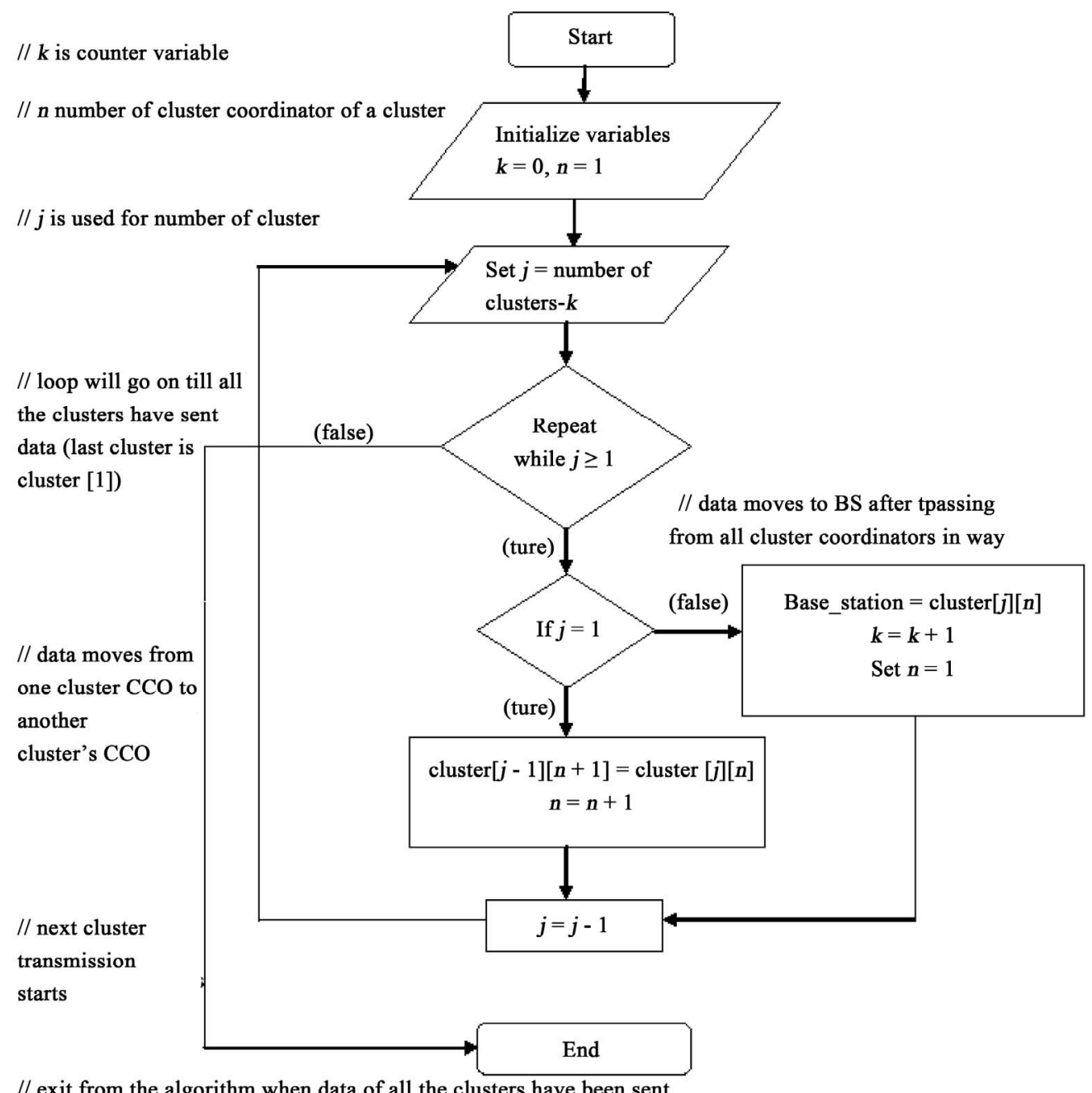

Figure 2. Flow chart of data transmission algorithm.

the messages from all other nodes. $l$ is the length of the message and $n 1$ is the number of nodes in one cluster. The election energy consumed by the non cluster heads is derived (Equation (5)) through Equations (2) and (3) is expressed as:

$$
\text { Enonch_elec }=k \cdot l\left(E e+E_{b f}\right)+l\left(E e+E s \cdot d^{2}\right)
$$

The first part of Equation (5) shows the energy consumed in receiving the messages from the $\mathrm{k}$ cluster heads and in new protocol the cluster heads are 10. And the second part shows the energy consumed in sending the acknowledgement to the cluster head by the nodes. This election will be done only once in starting of the round so the energy consumed is divided among all the clusters to know the energy consumption by one cluster as done in Equation (6) evaluated through Equations (2)-(5). When all the nodes will transfer their data then again the election process will done for the next round.

$$
\text { Et_elec }=(\text { Ech_elec }+ \text { Enonch_elec }) / k
$$

2) Transfer phase: In the transfer phase firstly the data is aggregated by the nodes on the respective cluster heads of each cluster and as the data travels through the clustercoordinators to reach the BS so energy consumed by the cluster head and the non cluster head in this phase is expressed by Equations (7) and (8).

$$
\begin{gathered}
\text { Ech_transfer }=l\left(E e+E s \cdot d^{2}\right)+n 1 \cdot l\left(E e+E_{b f}\right) \\
\text { Enonch_transfer }=l\left(E e+E s \cdot d^{2}\right)
\end{gathered}
$$

The Equation (7) shows the energy consumed by cluster head in transferring the data and in receiving the data from all the nodes. Equation (8) elaborates energy consumed by the non cluster head in sending the data to the cluster heads.

\section{Simulation Results and Discussion}

Based on the analytical work described in Section 5 extensive simulations are carried out in MATLAB. With 
the help of the mathematical model described in Section 3 various new equations have been derived and used in the simulations to get the results. The Equations (4)-(6) are being used to compute the energy consumed in election phase. Then it is compared with LEACH [2] and the Energy Efficient Hierarchical cluster based routing protocol (HCR) [3]. It can also be compared with the others protocols which are using the concept used in LEACH [2] and HCR [3] in which all clusters send their data send data directly to the base stations with the help of their own cluster head. New protocol proved to be better than others because of the following reasons:

1) EEICCP uses 10 clusters for the 1055 nodes which are very less than the LEACH [2] and the HCR [3]. Both conventional protocols has proved that the energy will be reduced when their will be more clusters. But this is not true. If the cluster head in both of them are sending the data to the base station by using multi path fading then the distance covered by all the cluster heads will be long and the path loss will be $d^{4}$. For 1055 nodes the cluster heads in HCR and LEACH are 50. It means $50 \mathrm{CHs}$ are sending their data by having the path loss component of $d^{4}$. If there will be more cluster heads, there will be more $d^{4}$ transmissions and the energy consumption will increase. But in proposed method the cluster heads and the cluster co-coordinators which are 55 all in number and the data transmission suffers from $d^{2}$ path loss because data is sent by using free space model and which is very less than the both protocols HCR and LEACH.

2) In the HCR in one round six iterations are performed calculated by $n / \mathrm{km}$ ( $n$ nodes, $m$ associates, $k$ clusters) and election of $\mathrm{CHs}$ is done for six times. But in proposed protocol election of CHs is done for one time in one round so energy consumption is reduced in election phase from $0.25-0.025 \mathrm{~J}$ (HCR) to $0.025-0.010 \mathrm{~J}$ (EEICCP) as shown in Figure 3.

3) Thirdly the associates of the clusters in the HCR [10] will sleep when they will be elected and they will be involved after sometime when again the election process starts i.e. before the end of one round they will wake and sleep for six times. But in EEICCP at the start of the protocol all the cluster heads and the cluster co-coordinators have to be alert but after the transmission by the $\mathrm{CH}$ of respective cluster $\mathrm{CH}$ and $\mathrm{CCO}$ of cluster can sleep for the entire period of the round. The cluster heads and the coordinators of the upper level sleep after aggregating the data until the data of the first transmitting cluster is transferred. So energy savings are high in this case. Because not only the cluster co-coordinators but the cluster heads also sleep to save the energy.

The energy consumption comparison is made for different phases with HCR [3] and LEACH [2], and found that EEICCP protocol performs better than both, Figures 3-7 show the validation of proposed strategy of the new

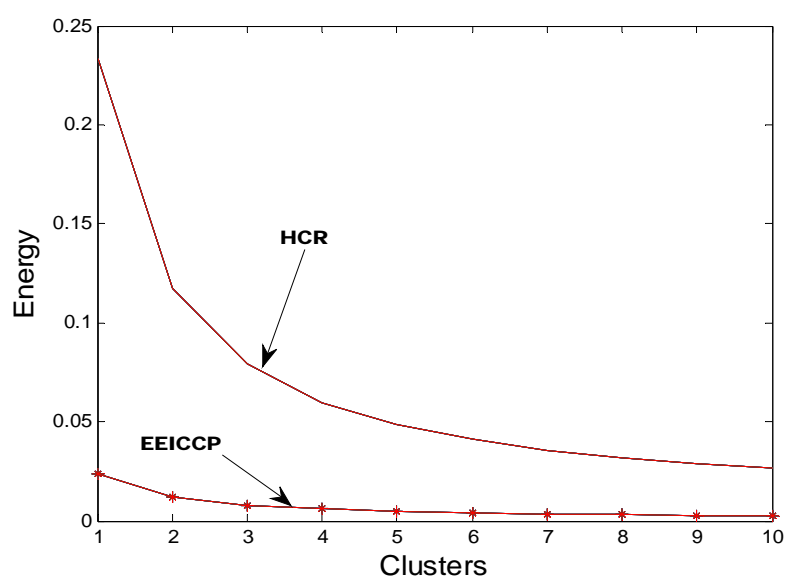

Figure 3. The energy consumed in election phase.

protocol. Election is done only one time in proposed work at the start of the round that is why energy consumed in election phase (Figure 3) can be uniformly divided into clusters by Ech_elec/ $k$ and Enonch_elec/ $k$. But in the HCR the election will be done for $\mathrm{n} / \mathrm{km}$ times in one round ( $n$ nodes, $k$ cluster heads and $m$ associates). Comparison among protocols (LEACH, HCR, EEICCP) shows that energy consumption in EEICCP protocol is reduced. For $k$ (10 in number) clusters start energy consumption also shows that the energy in the initial phase of EEICCP is less than that of the HCR and the LEACH. The start energy is calculated by the energy consumed in one iteration i.e. in the transfer phase energy consumption by $\mathrm{CH}$ and Non-CH for transmitting the data, in the election phase by all the cluster head nodes and the normal nodes for sending and receiving messages to elect CHs. This iteration energy is divided among all the cluster heads and the normal nodes which prove the validation of EEICCP protocol (Figure 4).

The transfer energy (Figure 5) of the cluster head includes the energy consumed in sending the data to the base station and in receiving the data from the other nodes. The transfer energy of the non cluster head nodes includes the energy consumed in sending the data to the cluster head. The methodology adopted is different from already developed protocols [1-4,6,8-10] and [15,16] because one cluster which is close to the base station have the cluster co-coordinators for all the clusters (Figure 1) which finally transmits data to the base station that is why path loss exponent is reduced. As data comes through the other cluster co-coordinators so calculation of not only the transfer phase is done for the single cluster head and its nodes but also the energy consumed by all the cluster heads and the cluster co-coordinators coming in the way to see how much energy is consumed (Figure 6).

For the 10 cluster heads and 30 cluster coordinators in HCR energy consumption is $0.030 \mathrm{~J}$ and for 10 cluster 


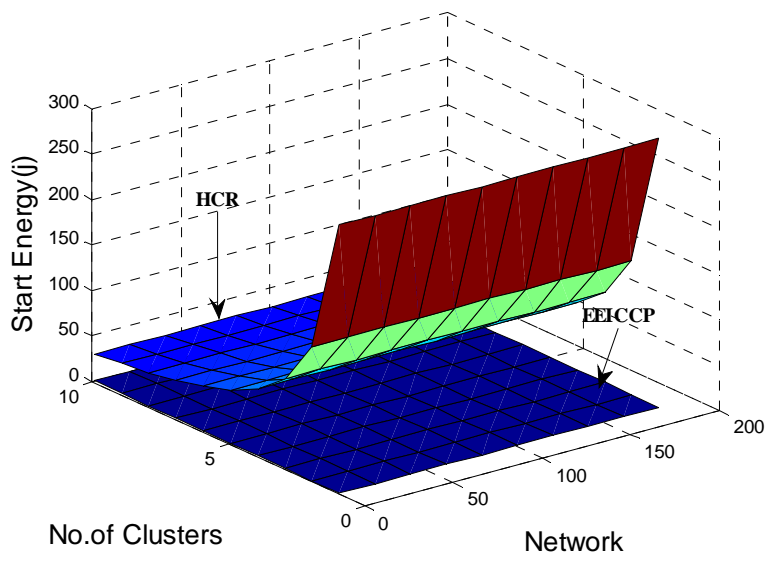

Figure 4. Start energy consumed per iteration.

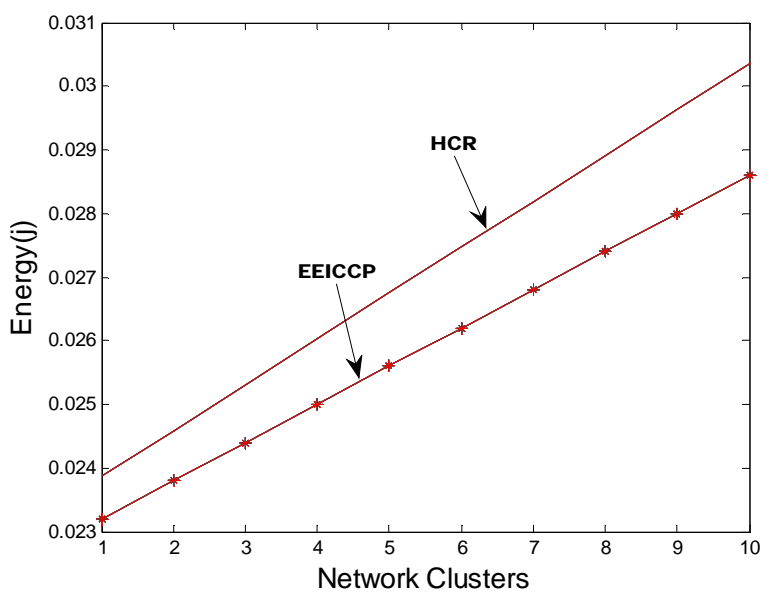

Figure 5. The transfer phase for the $k$ cluster heads and the non-cluster heads.

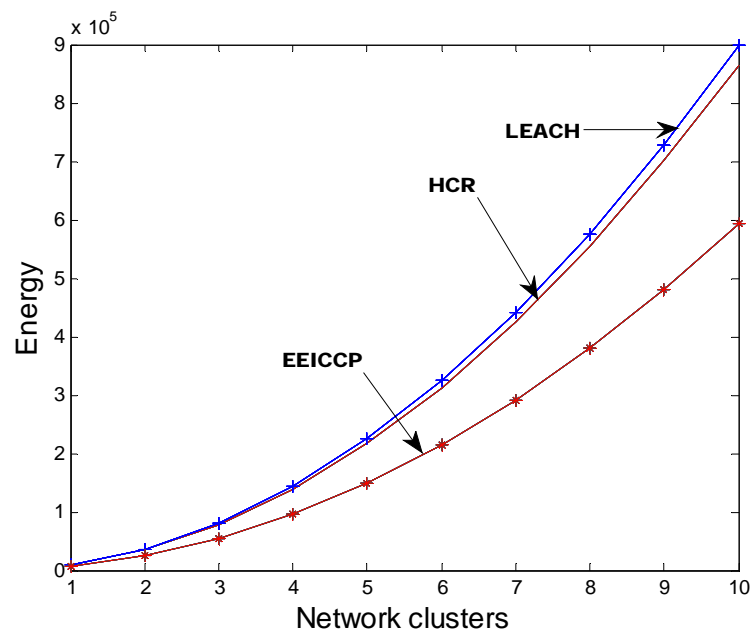

Figure 6. Total energy consumption for sending all the data of all the nodes.

heads and 45 cluster coordinators in the EEICCP it is $0.028 \mathrm{~J}$ approx (Figure 5). In this protocol cluster cocoordinators vary according to the depth of each cluster measured on the basis of received signal strength. It is observed that there is a greater variation of energy consumption in one iteration in LEACH [2] and HCR [3] but it remains constant for all the clusters in EEICCP (Figure 7) which proves that energy consumption is uniform in new protocol and hence leads to the long network lifetime of WSN.

The total energy consumption includes the energy of all the cluster heads, normal nodes and the cluster cocoordinators (Figure 6). In EEICCP the energy consumption is less when the cluster heads are less and is increased by increasing the cluster heads for the same number of nodes which shows contrast results than HCR and LEACH which have proved the energy consumption is more for less number of clusters and less for more clusters. In the HCR and LEACH computation is needed to find out the optimal number of cluster heads but this computation is not required in EEICCP protocol which decreases the complexity.

A simple strategy is developed by proposed protocol, if there are two clusters then the cluster nearest to the base station will have two clusters heads and the cluster below the upper cluster will have only one and so on. In the Table 2 it can be seen that in EEICCP (Figures 4-7) the energy consumption is very less as compared to the LEACH [2] and HCR [3]. So it can be said that new protocol serves better than early developed protocols discussed in this paper and it is the new research strategy which tries to the solve the main problem of battery powered sensors and that is energy constraint. Even for the higher densities (with 2210 nodes), this protocol consume less energy than LEACH and HCR as can be seen in Figure 8. But one problem with increasing number of

Table 2. Comparison of Energy consumption by HCR, LEACH and EEICCP.

\begin{tabular}{|c|c|c|c|c|}
\hline Phase & $\begin{array}{c}\text { EEICCP } \\
\text { Energy } \\
\text { (Joules) }\end{array}$ & $\begin{array}{c}\text { HCR } \\
\text { Energy } \\
\text { (Joules) }\end{array}$ & $\begin{array}{c}\text { LEACH } \\
\text { Energy } \\
\text { (Joules) }\end{array}$ & Remarks \\
\hline $\begin{array}{l}\text { Start energy } \\
\text { consumption } \\
\text { per iteration }\end{array}$ & $0.252-0.232$ & $300-35$ & $\begin{array}{c}\text { Same as } \\
\text { HCR }\end{array}$ & $\begin{array}{l}\text { A greater } \\
\text { variation of } \\
\text { energy } \\
\text { consumption in } \\
\text { HCR and } \\
\text { LEACH but } \\
\text { uniform in } \\
\text { EEICCP }\end{array}$ \\
\hline $\begin{array}{c}\text { Energy } \\
\text { consumption } \\
\text { in one } \\
\text { iteration }\end{array}$ & $\begin{array}{l}0.8201 \text { - } \\
0.8203\end{array}$ & $\begin{array}{l}2.4 \times 10^{4}- \\
0.24 \times 10^{4}\end{array}$ & $\begin{array}{l}2.5 \times 10^{4}- \\
0.26 \times 10^{4}\end{array}$ & $\begin{array}{l}\text { Same results } \\
\text { found in this } \\
\text { phase as in the } \\
\text { start energy } \\
\text { consumption }\end{array}$ \\
\hline $\begin{array}{l}\text { Total energy } \\
\text { consumption } \\
\text { for all the } \\
\text { nodes for } \\
\text { sending } \\
\text { all the data }\end{array}$ & $\begin{array}{c}0.1 \times 10^{5}- \\
6 \times 10^{5}\end{array}$ & $\begin{array}{c}0.2 \times 10^{5}- \\
8.6 \times 10^{5}\end{array}$ & $\begin{array}{c}0.2 \times 10^{5}- \\
9 \times 10^{5}\end{array}$ & $\begin{array}{c}\text { Energy } \\
\text { reduction in } \\
\text { EEICCP proves } \\
\text { its contribution } \\
\text { in long network } \\
\text { lifetime of WSN }\end{array}$ \\
\hline
\end{tabular}




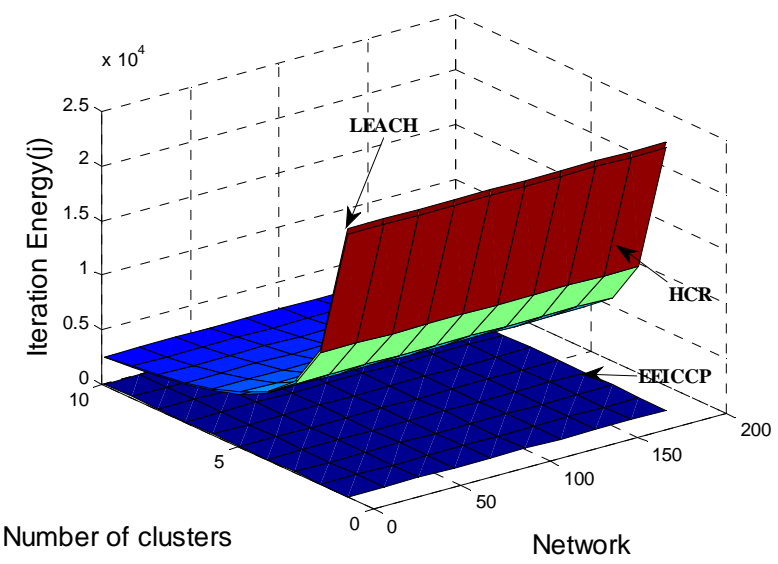

Figure 7. Energy consumption in one iteration.

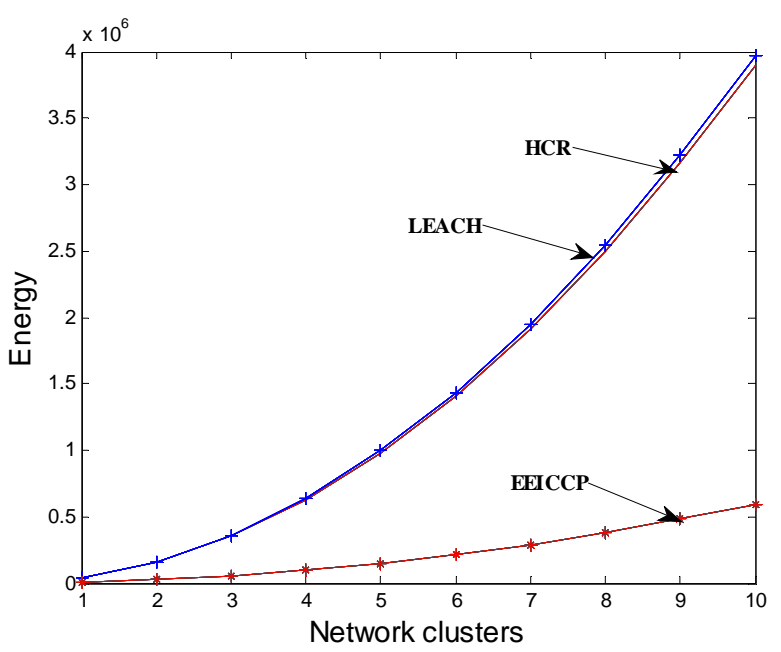

Figure 8. Total energy consumption for sending all the data of all the nodes (2210).

nodes is that the number clusters will increase. If we do not increase the number of clusters but increase the number of nodes then cluster size will increase so the power consumption of CCO will increase due to the processing of large number of nodes. As in the Figure 8, 2210 nodes are taken but clusters are 10 . But if 20 clusters are lay down then both $\mathrm{CH}$ and $\mathrm{CCO}$ will be needed 210 in EEICCP according to approach used which will increase energy consumption. This problem can be handled by dividing the clusters again in small clusters (clusters within clusters) and which will be considered in future work.

\section{Conclusion}

In this paper, new multi hop clustering based routing protocol EEICCP has been described, which minimizes the energy usage and further increases the network lifetime by uniform distributing load of energy among all the sensor nodes. EEICCP outperforms conventional protocols that send data directly to the BS through their re- spective CHs. Dividing the network into layers of clusters has been proved to be a good approach in reducing the energy to a great extent. Each node has the equal responsibility of receiving data from all other nodes in the cluster and to transmit the aggregating signal to the base station. Simulations show that EEICCP reduces start energy 151 times than both HCR and LEACH, energy of one iteration by $0.2926 \times 10^{4}$ times than HCR and 0.3169 $\times 10^{4}$ times than LEACH. Thus total energy reduction is 43\% than HCR and 50\% than LEACH. Simulations have been carried out in MATLAB that helped us to exploit the benefits of the propagation channels for longevity of the energy constrained network.

\section{REFERENCES}

[1] S. I. Matta and A. Bestavros, "SEP: A Stable Election Protocol for Clustered Heterogeneous Wireless Sensor Networks,” Technical Report BUCS-TR-2004-022G. http://open.bu.edu/xmlui/bitstream/handle/2144/1548/200 4-022-sep.pdf?sequence $=1$

[2] W. Heinzelman, A. Chandrakasan and H. Balakrishnan, "Energy-Efficient Communication Protocol for Wireless Microsensor Networks," Proceedings of the 33rd Hawaii International Conference on System Sciences, 4-7 January 2000.

[3] S. Hussain and A. W. Matin, "Energy Efficient Hierarchical Cluster-Based Routing for Wireless Sensor Networks,” Jodrey School of Computer Science Acadia University Wolfville, Nova Scotia, 2005, pp. 1-33.

http://cs.acadiau.ca/research/technical_reports/files/tr-200 5-011.pdf

[4] K. Ghosh, P. P. Bhattacharya and P. Das, "Effect of Multipath Fading and Propagation Environment on Performance of a Fermat Point Based Energy Efficient Geocast Routing Protocol,” International Journal of Wireless \& Mobile Networks, Vol. 4, No. 1, 2012, pp. 215-224. doi:10.5121/ijwmn.2012.4116

[5] H. Shpungin, "Feasible Capacity of Distributed Beamforming in Multi-Hop Wireless Sensor Networks," Mobile Adhoc and Sensor Systems (MASS), IEEE 8th International Conference, Valencia, 17-22 October 2011, pp. 19-24.

[6] G. Chen, C. Li, M. Ye and J. Wu, "An Unequal ClusterBased Routing Protocol in Wireless Sensor Networks," Wireless Networks, Vol. 15, No. 2, 2009, pp. 193-207.

[7] J. Zheng and A. Jamalipour, "Wireless Sensor Networks: A Networking Perspective,” John Wiley \& Sons, Hoboken, 2009.

[8] H. Chen and S. Megerian, "Cluster Sizing and Head Selection for Efficient Data Aggregation and Routing in Sensor Networks," IEEE Wireless Communications and Networking Conference, Las Vegas, 3-6 April 2006, pp. 2318-2323.

[9] S. Soro and W. B. Heinzelman, "Cluster Head Election Techniques for Coverage Preservation in Wireless Sensor Networks,” Ad Hoc Networks, Vol. 7, No. 5, 2009, pp. 
955-972.

[10] M. Ye, C. Li, G. Chen and J. Wu, "EECS: An Energy Efficient Clustering Scheme in Wireless Sensor Networks," 24th IEEE International Performance, Computing, and Communications Conference, 7-9 April 2005, pp. 535-540.

[11] G. Xin, W. H. Yang and B. De Gang, "EECHA-An Energy-Efficient Hierarchical Clustering Algorithm for Wireless Sensor Networks,” Information Technology Journal, Vol. 7, No. 2, 2008, pp. 245-252.

[12] K. Ghosh, S. Roy and P. K. Das and I. Min, “An Intelligent Fermat Point Based efficient Geographic Packet Forwarding Technique for Wireless Sensor and Ad Hoc Networks," International Journal on Applications of Graph Theory in Wireless Adhoc Networks and Sensor
Networks (GRAPH-HOC), Vol. 2, No. 2, 2010, pp. 34-44.

[13] T. Rappaport, "Wireless Communications: Principles \& Practice. Englewood Cliffs,” Prentice-Hall, Upper Saddle River, 1996.

[14] G. Andrea, "Wireless Communications,” 2005.

[15] N. Vlajic and D. Xia, "Wireless Sensor Networks: To Cluster or Not To Cluster?” Proceedings of the 2006 International Symposium on a World of Wireless, Mobile and Multimedia Networks, Buffalo-Niagara Falls, 2006, p. 268.

[16] O. Younis and S. Fahmy, "HEED: A Hybrid, EnergyEfficient, Distributed Clustering Approach for Ad-hoc Sensor Networks," IEEE Transaction on Mobile Computing, Vol. 3, No. 4, 2004, pp. 366-379. 